\title{
Clinical Investigation of Efficacy of Albumin Bound Paclitaxel plus Platinum Compounds as First-line Chemotherapy for Stage III/IV Squamous Non-small Cell Lung Cancer
}

\author{
Ying Fang, Li Wang, Guo-Hao Xia, Mei-Qi Shi*
}

\begin{abstract}
Objective: To observe the efficacy and toxicity of nanoparticle albumin bound paclitaxel (nab-paclitaxel) plus platinum agent (cisplatin or carboplatin) as first line treatment for stage III/IV squamous non-small-cell lung cancer (NSCLC). Methods: Forty chemotherapy naive patients with stage III/IV squamous NSCLC received nab-paclitaxel $125 \mathrm{mg} / \mathrm{m}^{2}$ on day 1 and day 8 , cisplatin $75 \mathrm{mg} / \mathrm{m}^{2}$ on day 1 , carboplatin area under the concentration-time curve of $5(\mathrm{AUC}=5)$ on day 1 . One cycle of treatment was 3 weeks, and at least two were completed in each case. Results: Of the 40 patients who participated in the study, 25 achieved partial responses $(\mathrm{PR}), 12$ reached a stage of stable disease (SD), and 3 suffered progressive disease (PD). The overall response rate (ORR) was $62.5 \%$ and the disease control rate (DCR) was $92.5 \%$. Of the 20 patients without surgery or radiotherapy, 10 achieved $\mathrm{PR}, 7$ reached a stage of SD, and 3 PD. The ORR was $50.0 \%$ and the DCR was $85.0 \%$. The median progression-free survival time (PFS) of patients without surgery or radiotherapy was 5.0 months. Of the 20 patients receiving surgery or radiotherapy, 15 had PR and $5 \mathrm{p}$ had SD, with an ORR of $75.0 \%$ and a DCR of $85.0 \%$. Specifically, the DDP arm demonstrated a significantly higher ORR than the CBP arm (100\% vs $54.5 \%, P<0.05)$. Common treatment related adverse events were myelosuppression, gastrointestinal response, baldness and neurotoxicity, most of which were grade 1 to 2. Conclusion: Nab-paclitaxel plus platinum agent (cisplatin or carboplatin) is effective as a first-line chemotheraphy for stage III/IV squamous NSCLC, and its adverse effects are tolerable.
\end{abstract}

Keywords: Albumin bound paclitaxel - cisplatin - carboplatin - squamous non-small cell lung cancer

Asian Pac J Cancer Prev, 15 (17), 7453-7457

\section{Introduction}

Recently, great progression have been made on the research of pulmonary adenocarcinoma. Pemetrexed, a third-generation chemotherapeutic agent, has showed its favorable efficacy and tolerable toxicity both in first-line, second-line and maintenance treatment of pulmonary adenocarcinoma (Hanna et al., 2004; Scagliotti et al., 2008; Ciuleanu et a1., 2009), so the combination chemotherapy of pemetrexed and platinum compounds is recommended as first-line standard therapy for advanced non-small cell lung cancer (NSCLC) by NCCN. Targeted drugs, such as bevacizumab, combined with chemotherapy, are also quitely effective treatment for pulmonary adenocarcinoma (Barlesi et al., 2013). There are various therapeutic treatments of targeted EGFR-TKI inhibiter owing to the 50 60\% EGFR mutation rate in Asiatic pulmonary adenocarcinoma patients (Shi et al., 2014). In contrast, there is no breakthrough in treatment of pulmonary adenocarcinoma and the treatment options are also limited. Nanoparticle albumin bound paclitaxel (nab-paclitaxel), a new type of paclitaxel praeparatum, has many advantages like high efficiency, low toxicity and convenience. Our department applied nanoparticle albumin bound paclitaxel (nab-paclitaxel) combinded platinum agents (cisplatin, DPP or carboplatin, CBP) as first-line treatment in 40 lung squamous carcinoma patients between February, 2011 to October, 2013, and the results are as follows.

\section{Materials and Methods}

\section{Clinical data}

Eligible adults had histologically/cytologically confirmed nonresectable stage III or IV squamous carcinoma from February 2011 to October 2013. All patients were naive cases, including 36 males and 4 females, aged between 30 to 79 years, the median age was 61.26 cases in stage III including 24 cases in III A period and 2 cases in III B ; 14 cases in IV ; 19 cases receiving nanoparticle albumin bound paclitaxel plus cisplatin, 21 cases in combination with carboplatin. All patients were 
Table 1. Baseline Characteristics of 40 Patients

\begin{tabular}{lrc}
\hline Clinical feature & $\mathrm{N}$ & Percentage \\
\hline Sex & & \\
$\quad$ Male & 36 & $90.0 \%$ \\
$\quad$ Female & 4 & $10.0 \%$ \\
Age & & \\
$\quad$ Median & 61 & \\
$\quad<65$ & 27 & $67.5 \%$ \\
$\quad \geq 65$ & 13 & $32.5 \%$ \\
ECOG scores & & \\
$\quad 0 \sim 1$ & 37 & $92.5 \%$ \\
$1-3$ & 3 & $7.5 \%$ \\
Clinical stage & & \\
$\quad$ Stage III & 26 & $65.0 \%$ \\
$\quad$ Stage III A & 24 & $60.0 \%$ \\
$\quad$ Stage III B & 2 & $5.0 \%$ \\
$\quad$ Stage IV & 14 & $35.00 \%$ \\
Therapeutic schedule & & \\
$\quad$ nab-PC+DDP & 19 & $47.5 \%$ \\
$\quad$ nab-PC+CBP & 21 & $52.5 \%$ \\
\hline
\end{tabular}

confirmed the presence of measurable lesions by CT scan and the Eastern Cooperative Oncology Group (ECOG) score was $0 \sim 2$, and a life expectancy of more than 3 months. Their routine blood test and electrocardiogram were generally normal before treatment, meanwhile liver and kidney functions $\leq 1.5$ times the normal range. All patients signed consent form before treatment. Table 1 gives general situation of 40 cases.

\section{Methods}

Albumin-bound paclitaxel was used at a dose of 125 $\mathrm{mg} / \mathrm{m}^{2}$ (injection for over 30 minutes). The drug was administered on a day 1 and day 8 schedule every three weeks. Cisplatin was administered at $75 \mathrm{mg} / \mathrm{m}^{2}$ on day 1 every three weeks.Carboplatin was administered at an area under the curve (AUC) of 6 calculated according to the Calvert formula on day 1 every three weeks. The efficacy was evaluated every two cycles. Hematologic and imaging examinations were routinely performed during the medication. The original protocol would be kept for 2 4 cycles if it was effective.

\section{Assessment of efficacy and adverse reactions}

The objective efficacy was evaluated using the Response Evaluation Criteria in Solid Tumors (RECIST) 1.1. The observation indicators included complete remission (CR), partial response (PR), stable disease (SD), disease progression (PD), objective response rate (ORR), and disease control rate (DCR). Progression-free survival (PFS) is defined as the time from first medication to the first objective progression of disease. The evaluation of adverse reactions was based on the NCI Common Toxicity Criteria (CTC) Version 3.0.

\section{Statistics analysis}

Statistical analysis was done by SPSS 13.0 software. Correlation between clinical features and curative effects was valued by using $\chi^{2}$ test. PFS was analyzed using Kaplan-Meier methods. A value of $p<0.05$ was considered to be statistically significant.
Table 2. Clinical Feature and Correlation of Curative Effect the of Nab-paclitaxel Plus Platinum Agent as First Line Treatment on Lung Squamous Carcinoma

\begin{tabular}{|c|c|c|c|c|c|c|c|c|}
\hline Clinical feature & $\mathrm{N}$ & PR & SD & PD & ORR & $P$ & DCR & $P$ \\
\hline \multicolumn{6}{|l|}{ Sex } & 0.586 & & 0.548 \\
\hline Male & 36 & 23 & 10 & 3 & $63.9 \%$ & \multicolumn{3}{|c|}{$91.7 \%$} \\
\hline Female & 4 & 2 & 2 & 0 & $50.0 \%$ & \multicolumn{3}{|c|}{$100 \%$} \\
\hline \multicolumn{6}{|l|}{ Age } & 0.433 & & 0.974 \\
\hline$<65$ & 27 & 18 & 7 & 2 & $69.2 \%$ & \multicolumn{3}{|c|}{$92.5 \%$} \\
\hline$\geq 65$ & 13 & 7 & 5 & 1 & $53.8 \%$ & \multicolumn{3}{|c|}{$92.3 \%$} \\
\hline \multicolumn{6}{|l|}{ Clinical stage } & \multicolumn{2}{|l|}{0.608} & \multirow{3}{*}{0.950} \\
\hline Stage III & 26 & 17 & 7 & 2 & $65.4 \%$ & & $92.3 \%$ & \\
\hline Stage IV & 14 & 8 & 5 & 1 & $57.1 \%$ & & $92.9 \%$ & \\
\hline \multicolumn{6}{|c|}{ Therapeutic schedule } & \multicolumn{2}{|l|}{0.165} & \multirow{3}{*}{0.489} \\
\hline Nab-PC+DDP & 19 & 14 & 3 & 2 & $73.7 \%$ & & $89.5 \%$ & \\
\hline $\mathrm{Nab}-\mathrm{PC}+\mathrm{CBP}$ & 21 & 11 & 9 & 1 & $52.4 \%$ & & $95.2 \%$ & \\
\hline \multicolumn{6}{|l|}{ ECOG scores } & \multirow[t]{3}{*}{0.278} & & \multirow{3}{*}{0.077} \\
\hline $0 \sim 1$ & 37 & 24 & 11 & 2 & $64.9 \%$ & & $94.6 \%$ & \\
\hline 2 & 3 & 1 & 1 & 1 & $33.3 \%$ & & $66.7 \%$ & \\
\hline
\end{tabular}

Table 3. Analysis of Curative Effect in Subgroup without Surgery or Radiotherapy

\begin{tabular}{|c|c|c|c|c|c|c|c|c|}
\hline $\begin{array}{l}\text { Group without } \\
\text { surgery or radiother }\end{array}$ & $\begin{array}{l}\mathrm{N} \\
\text { rapy }\end{array}$ & PR & SD & PD ORR & $P$ & DCR & $P \mathrm{P}$ & $\mathrm{FS}(\mathrm{m}$ \\
\hline Stage III & 7 & 2 & 3 & $28.6 \%$ & 0.160 & $71.4 \%$ & 0.212 & 4.3 \\
\hline Stage IV & 13 & 8 & 4 & $61.5 \%$ & & $92.3 \%$ & & 5.3 \\
\hline Group plusDDP & 10 & 5 & 3 & $50.0 \%$ & 1.000 & $80.0 \%$ & 0.531 & 4.5 \\
\hline Group plus CBP & 10 & 5 & 4 & $50.0 \%$ & & $90.0 \%$ & & 5.4 \\
\hline Total & 20 & 10 & 7 & $50.0 \%$ & & $85.0 \%$ & & 5.0 \\
\hline
\end{tabular}

\section{Results}

Short-term efficacy

All patients were eligible for efficacy evaluation. A total of 137 cycles of treatment was delivered overall, with a median number of cycles per patient of 3 (range, 1 to 6). There were 25 cases of PR (62.5\%), 12 cases of SD (30.0\%), 3 cases of PD (7.5\%), and ORR 62.5\% (25/40), DCR92.5\% (37/40). There were 14 cases of PR $(73.7 \%)$, 3 cases of SD (15.8\%), 2 cases of PD (10.5\%), ORR $73.7 \%$ (14/19), DCR89.5\% (17/19)in the group which 19 patients received treatment plus cisplatin. There were 11 cases of PR (62.5\%), 9 cases of SD (30.0\%), 1 cases of PD (7.5\%), ORR52.4\% (11/21), DCR95.2\% (20/21) in the group which 21 patients received treatment plus carboplatin. 15 patients received radiotherapy and 4 patients received surgery after the 26 patients on stage III received 2 3 cycles of chemotherapy. 7 patients gave up radiotherapy or surgery treatment because of their poor pulmonary function, multiple primary diseases and intolerance in which there were 2 cases of PR (28.6\%), 3 cases of SD (42.9\%) and 2 cases of PD (28.6\%). 1 case with metastasis of sinistro-adrenal gland in the 14 patients on stage IV was evaluated PR and received treatment of surgery according to multi-disciplinary assessment after 2 3 cycles of chemotherapy. There were 8 cases of PR (61.5\%), 4 cases of SD (30.8\%) and 1 case of PD $(7.7 \%)$ in the 13 patients who neither received surgery nor radiotherapy. In the whole group, 20 patients neither received surgery nor radiotherapy, in which there were 15 cases of PR (75.0\%), 5 cases of SD $(25.0 \%)$ and 0 case of PD, ORR75.0\% (15/20), DCR100\% (20/20); 9 cases received treatment plus cisplatin in which there were 9 cases of PR (100\%), 0 case of SD, 0 case of PD, ORR 
Table 4. Analysis of Curative Effect in Subgroup with Surgery or Radiotherapy

\begin{tabular}{lrrrrrr}
\hline $\begin{array}{l}\text { Group with surgery } \\
\text { or radiotherapy }\end{array}$ & $\mathrm{N}$ & PR & SD & PD & ORR & $P$ \\
\hline Stage III & 19 & 14 & 5 & 0 & $73.7 \%$ & 0.544 \\
Stage IV & 1 & 1 & 0 & 0 & $100 \%$ & \\
Group plus DDP & 9 & 9 & 0 & 0 & $100 \%$ & 0.038 \\
Group plus CBP & 11 & 6 & 5 & 0 & $54.5 \%$ & \\
Total & 20 & 15 & 5 & 0 & $100 \%$ & \\
\hline
\end{tabular}

Table 5. Adverse Effects of Nab-paclitaxel Plus Platinum Agent

\begin{tabular}{lrrr}
\hline Adverse effects & \multicolumn{3}{c}{ WHO grade } \\
\cline { 2 - 4 } & I/II (\%) & \multicolumn{1}{c}{ III (\%) } & IV (\%) \\
\hline Leukopenia & $14(35.0 \%)$ & $8(20.0 \%)$ & $2(5.0 \%)$ \\
Thrombocytopenia & $5(12.5 \%)$ & $2(5.0 \%)$ & $2(5.0 \%)$ \\
Baldness & $15(37.5 \%)$ & $9(22.5 \%)$ & $0(0.0 \%)$ \\
Neurotoxicity & $20(50.0 \%)$ & $3(7.5 \%)$ & $0(0.0 \%)$ \\
Nauseated, vomiting & $21(52.5 \%)$ & $4(12.5 \%)$ & $0(0.0 \%)$ \\
Liver function damage & $4(10.0 \%)$ & $0(0.0 \%)$ & $0(0.0 \%)$ \\
Renal function damage & $2(5.0 \%)$ & $0(0.0 \%)$ & $0(0.0 \%)$ \\
\hline
\end{tabular}

$100.0 \%(9 / 9)$, DCR $100 \%(9 / 9)$ and 11 cases received treatment plus carboplatin in which there were 6 cases of PR (54.5\%), 5 cases of SD (45.5\%), 0 case of PD, ORR $54.5 \%$ (6/11), DCR 100\% (11/11) (Table 2 5).

\section{Survival analysis}

We kept following up by clinic service or telephone from the beginning of treatment to October 31st, 2013. PFS of the 20 patients who had no surgery or radiotherapy was $1.4 \sim 17.9$ months, and median-PFS was 5 months in which PFS of patients on stage III was 4.3 months and 5.3 of stage IV. PFS of patients received cisplatin was 4.5 months and that received carboplatin was 5.4 months. The PFS of 19 cases on stage III and 1 case on stage IV was censored values because of their treatment of surgery or radiotherapy (Table 4, Figure 1 3).

\section{Adverse effects}

Adverse effects of nanoparticle albumin bound paclitaxel (nab-paclitaxel) plus platinum agent (cisplatin or carboplatin) was myelosuppression, gastrointestinal response, badlness and neurotoxicity. I/II degree leukopenia were $14(35.0 \%)$, III degree 8 (20.0\%) and IV degree $2(5.0 \%)$. I/II degree decreased platelet count were $5(12.5 \%)$, III degree $9(22.5 \%)$ and IV degree 2 (5.0\%). I/II degree hair loss were 15 (37.5\%)and III degree were $9(22.5 \%)$. I/II degree periphery neurotoxicity were $20(50.0 \%)$ and III degree were $3(7.5 \%)$. I/II degree gastrointestinal reaction were $21(52.5 \%)$ and III degree were $4(10.0 \%)$. I/II degree liver damage were $4(10.0 \%)$. I/II degree kidney damage were 2 (5.0\%).

\section{Discussion}

Paclitaxel, with broad-spectrum antitumor activity, is approved for the treatment of advanced non-small-cell lung cancer (Li et al., 2013; Natukula et al., 2013; Gurses et al., 2013; Ozdemir et al., 2013; Cui et al., 2014). With the vector of albumin, it can reduce risk of hypersensitivity

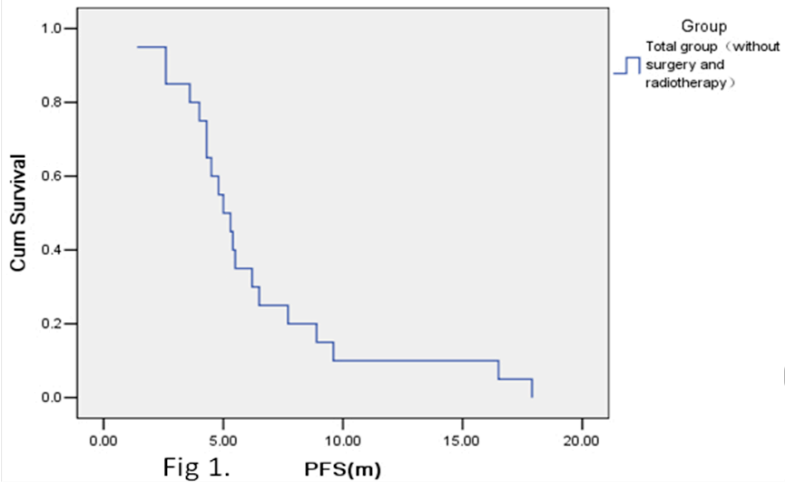

Figure 1. Progression-free Survival (PFS) of Allpatients without Surgery or Radiotherapy. The median PFS were 5 months

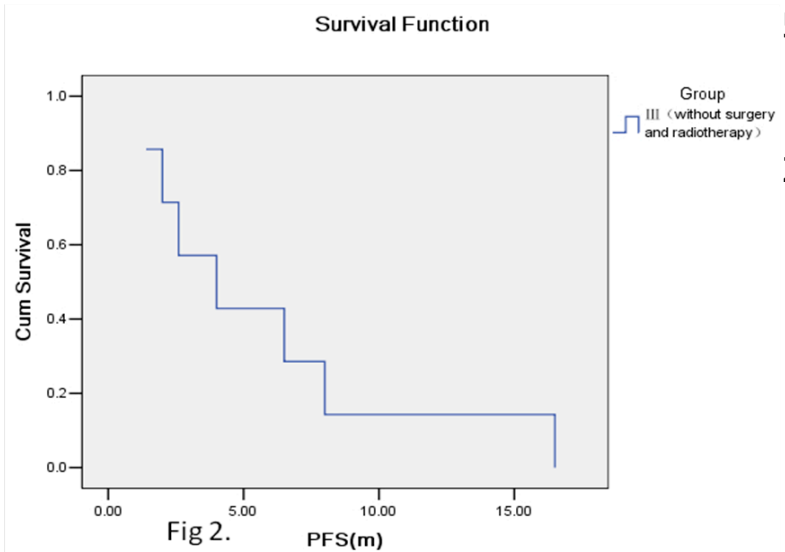

Figure 2. Progression-free Survival (PFS) of Stage III Patients without Surgery or Radiotherapy. The median PFS were 4.3 months

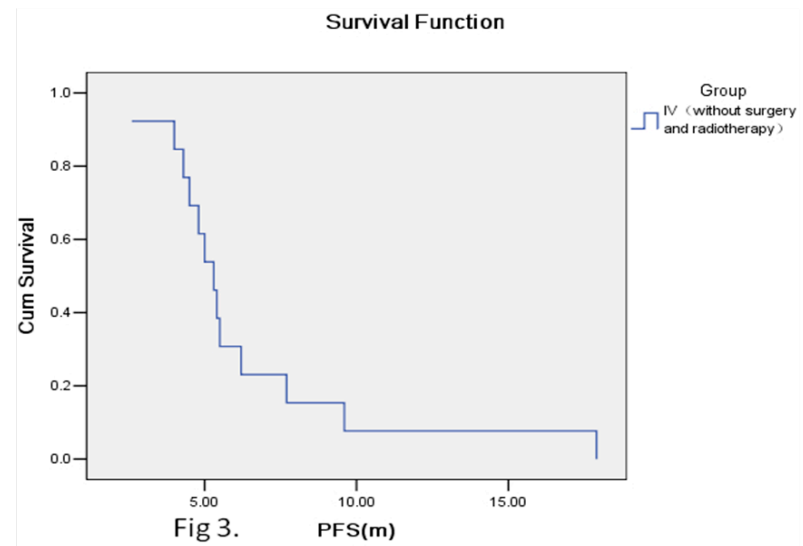

Figure 3. Progression-free Survival (PFS) of Stage IV Patients without Surgery or Radiotherapy. The median PFS were 5.3 months

reactions and blood toxicity induced by organic solvent. It exploits the natural properties of album into increase of uptake and causes accumulation of paclitaxel in the tumor through gp 60-mediated endothelial transcytosis (Aapro et al., 2008), and interaction with the album inbinding protein SPARC (secreted protein, acidic and rich in cysteine). Nab-paclitaxel has greater antitumor activity with increased intratumoral concentrations (Trieu et al., 2006; Desai et al., 2009). Socinski et al. (2013) conducted a major international multicenter randomized phase III 
clinical study of nab-paclitaxel compared solvent-based paclitaxel. 1052 patients in the phase III/IV NSCLC was enrolled and randomly assigned to carboplatin and either nab-paclitaxel or solvent-based paclitaxel for the firstline treatment. the ORR was higher with nab- $P / \mathrm{C}$ versus sb-P/C ( $34 \%$ versus $25 \%, P=0.005)$. further histologic subgroup analysis for squamous histology indicated the ORR was $67 \%$ higher with nab-paclitaxel $(41 \%)$ than solvent-based paclitaxel (24\%). There was no survival difference between nab-paclitaxel and solvent-based paclitaxel. This is the best efficacy of lung squamous carcinoma data reported in literatures. According to the analysis by investigator, the treatment with nab-paclitaxel demonstrated greater short-term efficacy with potentially increasing uptake and accumulation of paclitaxel in the tumor through gp60-CAV1 mediated endothelial transcytosis induced by abnormal higher expressions of CAV-1.

In this study we enrolled 40 patients with lung squamous carcinoma took nab-paclitaxel combined with cisplatin or carboplatin. The ORR was $62.5 \%$ and the DCR was $92.5 \%$. The ORR was up to $73.7 \%$ in patients with nab-paclitaxel combined with cisplatin, and was better than the data reported in the literature. In Socinski's study, the ORR was $44.4 \%$ in lung squamous carcinoma patients. The treatment of nab-paclitaxel showed better effective compared with solvent-based paclitaxel in western countries, for instance, the efficacy of the general third generation platinum-based two drugs combination chemotherapy was $17 \%$ to $22 \%$ ( Schiller et al., 2002). The reason for the efficacy in this study was superior to the results reported maybe related to the race. The previous research revealed the efficacy of chemotherapy or targeted therapy of East Asian patients was better than that on Western patients. The median PFS in the patients without surgery or radiotherapy was 5 months, the phase III patients 4.3 months and the phase IV patients 5.3 moths. The reason for the higher efficacy seemingly did not translate to the median PFS extention compared with 5.6 months median PFS reported in Socinski's study in lung squamous carcinoma, it is probably attributed to that the patients abandon the initial therapy without disease progression, such as, turned to surgery or radiotherapy. The continuity of chemotherapy was effected because that the patients who was older, with poor lung functions and suffering diseases could not receive the further surgery or radiotherapy. the prolong of median PFS was effected with some patients who was in phase IV and discontinued the therapy because of the economy or un-tolerance adverse drug reaction. The ORR in the treatment combined with cisplatin (100\%) was tremendously higher than that with carboplatin $(63.6 \%)$ in the patients with surgery or radiotherapy. The treatment of nab-paclitaxel plus platinum-based chemotherapy may be a optimal protocol for the patients with good physical situation and tolerance because that it could alleviate the tumor load rapidly and strengthen the efficacy of local tumor especially to make a chance of the surgery for the potential patients and maximized reduction of the volume of the tumor.

Finally, the nab-PC showed a better safety and tolerability with neutropenia, sensory nerve toxicity, the incidence of myalgia obviously reduced, except anemia and thrombocytopenia increased. Nab-PC was approved chemotherapy agent for the patients with advanced or metastatic NSCLC in the first-line treatment.

In general, the most common AEs were myelosuppression, gastrointestinal reaction and baldness, and secondary AEs neurovirulence. The most AEs were I/II degree, except 4 myelosuppression with IV degree which eased after symptomatic therapy. And there was no treatment-related deaths occurred. In sum, the nabpaclitaxel combined with cisplatin or carboplatin has a good tolerance for lung squamous carcinoma with the first-line treatment.

In summary, the combination of nab-paclitaxel and cisplatin or carboplatin indicated better short-term efficacy, less AEs, and well tolerated. The protocol of nab-paclitaxel combined with platinum-based make a chance for the treatment of the lung squamous carcinoma especially multidisciplinary synthetic therapy of locally advanced lung squamous carcinoma to improve the efficacy and prolong survival with currently less selectable chemotherapy and no precise efficacy of targeted-therapy. This study was a retrospective analysis with small sample size. The efficacy and toxicity of the treatment of nabpaclitaxel combined with platinum-based for Asian or Chinese patients needed a prospective, large sample, multi-center clinical study.

\section{References}

AaproMS, Minckwitz GV (2008). Molecular basis for the development of noveltaxanes in the treatment of metastatic breast cancer. Eur J Cancer Suppl, 6, 3-11.

Barlesi F, Scherpereel A, Rittmeyer A, et al. (2013). Randomized phase III trial of maintenance bevacizumab with or without pemetrexed after first-line induction with bevacizumab, cisplatin, and pemetrexed in advanced nonsquamous nonsmall-cell lung cancer: AVAPERL(MO22089). J Clin Oncol, 31, 3004-11.

Ciuleanu T, Brodowicz T, Zielinski C, et a1 (2009). Maintenance pemetrexed plus best supportive care versus placebo plus best supportive care for non-small-cell lung cancer, a randomised, double-blind, phase 3 study. Lancet, 374, 1432-40.

Cui L, Liu XX, Jiang Y, et al (2014)Phase II study on dose escalating schedule of paclitaxel concurrent with radiotherapy in treating patients with locally advanced non-small cell lung cancer. Asian Pac J Cancer Prev, 15, 1699-702.

Desai N, Trieu V, Damascelli B, etal. (2009). SPARC expression Correlates with tumor response to albumin-bound paclitaxel in headand neck cancerpatients. Transl Oncol, 2, 59-64.

Hanna N, Shepherd FA, Fossella FV, et a1 (2004). Randomized phase III trial of pemetrexed versus docetaxel in patients with non-small-cell lung cancer previously treated with chemotherapy. J Clin Oncol, 22, 1589-97.

Gurses N, Topcul M (2013). The effect of abraxane on cell kinetic parameters of HeLa cells. Asian Pac J Cancer Prev, 14, 4229-33.

Li XD, Han JC, Zhang YJ, et al (2013). Common variations of DNA repair genes are associated with response to platinumbased chemotherapy in NSCLCs. Asian Pac J Cancer Prev, 14, 145-8.

Natukula K, Jamil K, Pingali UR, et al (2013). Survival 

analysis in advanced non small cell lung cancer treated with platinum based chemotherapy in combination with paclitaxel, gemcitabine and etoposide. Asian Pac J Cancer Prev, 14, 4661-6.

Ozdemir O, Ozdemir P, Veral A, et al (2013). ERCC1 expression does not predict survival and treatment response in advanced stage non-small cell lung cancer cases treated with platinum based chemotherapy. Asian Pac J Cancer Prev, 14, 4679-83.

Scagliotti GV, Parikh P, von Pawel J, et a1 (2008). Phase III study comparing cisplatin plus gemcitabine with cisplatin plus pemetrexed in chemotherapy-naive patients with advanced-stage non-small-cell lung cancer. J Clin Oncol, 26, 3543-51.

Schiller JH, Harrington D, Belani CP, et al. Comparison of four chemotherapy regimens for advanced non-small-cell lung cancer. N Engl J Med, 346, 92-8.

Shi YK, Au JS, Thongprasert S, etal. (2014). A prospective, molecular epidemiology study of EGFR mutations in Asian patients with advanced non-small-cell lung cancer of adenocarcinoma histology (PIONEER). J Thorac Oncol, 9, 154-62.

Socinski MA, Langer CJ, Okamoto I, et al. (2002). Safety and efficacy of weekly nab-paclitaxel in combination with carboplatin as first-line therapy in elderly patients with advanced non-small-cell lung cancer. Ann Oncol, 24, 314-21.

Trieu V, Damascelli B, Soon-ShiongP, et al ( 2008). SPARC expressionin head and neck cancer correlates with tumor response to nanoparticle albumin-bound paclitaxel (nabpaclitaxel, ABI-007 Abraxane). Washington, DC, the 97th Annual Meetingof the American Association for Cancer Research (AACR), 2006. 